\title{
Melanopsin retinal ganglion cell loss and circadian dysfunction in Alzheimer's disease (Review)
}

\author{
RUIQI FENG ${ }^{1}$, LIJUAN LI ${ }^{2}$, HAIYAN YU ${ }^{3}$, MIN LIU $^{2}$ and WEI ZHAO ${ }^{4}$ \\ ${ }^{1}$ Department of Geriatrics, Yunnan Provincial Mental Hospital, Kunming, Yunnan 650223; ${ }^{2}$ Administrative \\ Office, School of Public Health, Dali University, Dali, Yunnan 671000; ${ }^{3}$ Department of Pharmacy, \\ Yunnan Provincial Mental Hospital, Kunming, Yunnan 650223; ${ }^{4}$ Department of Ophthalmology, \\ School of Clinical Medicine, Dali University, Dali, Yunnan 671000, P.R. China
}

Received December 4, 2015; Accepted March 2, 2016

DOI: $10.3892 / \mathrm{mmr} .2016 .4966$

\begin{abstract}
Alzheimer's disease affects 27 million individuals and is the most common cause of dementia worldwide. The pathology of Alzheimer's disease is primarily due to the $\beta$-amyloid deposits and neurofibrillary tangles. These deposits exist largely in the cerebral blood vessels, but have also been shown to exist in retinal vessels. A new class of cells that were recently identified, known as melanopsin-expressing retinal ganglion cells (mRGCs), are involved in the non-image forming functions of the eye. These functions include circadian activities such as temperature rhythms, melatonin release and rest-activity cycles. Circadian dysfunction has been investigated in many cases of Alzheimer's disease. In this review, we outline the current accepted Alzheimer's disease pathology, the role of mRCGs in optic neuropathies and the role of mRCGs, leading to circadian dysfunction, in Alzheimer's disease.
\end{abstract}

\section{Contents}

1. Introduction

2. Alzheimer's disease epidemiology

3. Alzheimer's disease pathology

4. Melanopsin-expressing RGCs

5. The mRGCs in visual defects

6. Role of mRGCs in migraines

7. Role of mRGCs in aging

8. Role of mRGCs in Alzheimer's disease patients

9. Changes in circadian rhythms in Alzheimer's disease patients

10. Conclusion

Correspondence to: Dr Lijuan Li, Administrative Office, School of Public Health, Dali University, No. 2 Xueren Road, Dali, Yunnan 671000, P.R. China

E-mail: 1i_lijuan1@163.com

Key words: melanopsin retinal ganglion cell, Alzheimer's disease, circadian dysfunction

\section{Introduction}

Alzheimer's disease, characterized by $\beta$-amyloid deposits and neurofibrillary tangles, is the most common type of dementia in the elderly population. The clinical duration is normally 8-10 years, and mortality often results due to secondary factors, such as pneumonia (1). When diagnosing the disease, pathologic findings of $\beta$-amyloid plaques and neurofibrillary tangles as well as the neuroimaging of gross cerebral cortical atrophy are the standard. The disease begins with memory loss and can include confusion, withdrawal, agitation, hallucinations and lack of judgement $(2,3)$.

Previous findings $(4,5)$ have shown that Alzheimer's disease patients have circadian dysfunction of melatonin, temperature rhythms and rest-activity. Additionally, use of actigraphy, a non-invasive procedure of monitoring rest-activity cycles, has shown an increased intradaily variability, reduced interdaily stability and amplitude of rest-activity rhythm in patients with Alzheimer's disease (6). The suprachiasmatic nucleus, which is a small region located in the hypothalamus and above the optic chiasm, is responsible for controlling the circadian rhythm. In patients with Alzheimer's disease, there was found to be an increase in neuron loss in the suprachiasmatic nucleus (7).

The pathology of Alzheimer's disease shows neurodegeneration in the brain, which includes retinal and optic nerve tissues. Tests such as optical coherence tomography confirmed that Alzheimer's disease leads to a decrease in retinal nerve fiber layer thickness. In addition, $\beta$-amyloid deposits in the retina of patients with Alzheimer's disease have been reported. Three different studies (8-10) identified that Alzheimer's disease patients exhibited diffuse, immature and mature $\beta$-amyloid deposits in the retinas. Therefore, the pathogenesis of Alzheimer's disease extends beyond cerebral blood vessels and includes the retina (11).

Newly identified melanopsin-expressing retinal ganglion cells (mRGCs) are photoreceptors that function in maintaining circadian rhythms, regulation of pupil size, sleep, alertness and other non-visual purposes of the eye. These cells comprise $1-2 \%$ of all RGCs. The mRGCs convey information to the brain through a monosynaptic neuronal pathway, known as the retinohypothalamic tract, which projects to 
the suprachiasmatic nucleus (Fig. 1). The role of mRGCs in different diseases has been investigated. A loss of mRGCs is associated with older age, as well as with diseases such as glaucoma. In this review, we outlined the latest findings on the new class of photoreceptors and their role in Alzheimer's disease pathology $(12,13)$.

\section{Alzheimer's disease epidemiology}

There are approximately 5 million individuals in the United States living with Alzheimer's disease, which is the fifth leading cause of mortality in the elderly population (14). Women are more likely to experience Alzheimer's disease, which may be due to longer life spans. The primary risk factor for developing Alzheimer's disease is age. Over $95 \%$ of the causes of Alzheimer's disease affect those over the age of 65 years. The prevalence of Alzheimer's disease is $10-30 \%$ in patients aged $>65$ years. The likelihood of developing Alzheimer's disease doubles every 5 years after the age of 65 . Individuals with familiar forms of Alzheimer's disease have an autosomal dominant mutation in one of the presenilin genes or the amyloid precursor protein $(A P P)$ gene. Other factors that may lead to Alzheimer's disease pathology are diabetes, hypertension, obesity, smoking and the presence of Alzheimer's disease in first-degree relatives (15).

\section{Alzheimer's disease pathology}

The mechanism for Alzheimer's disease pathology that is most widely accepted is the amyloid cascade hypothesis. Under normal conditions, the enzyme $\alpha$-secretase cleaves APP correctly, which allows the APP protein to fulfill its function (16). APP usually responds to cell injury. The amyloid cascade hypothesis states that under pathological conditions, APP is incorrectly cleaved by $\beta$ - and $\gamma$-secretase. Incorrect processing of the APP gene causes the production of a modified version of $\beta$-amyloid peptides, which leads to the formation of $\beta$-amyloid extracellular deposits (17). These plaques are generated in the brain parenchyma and in cerebral blood vessels. The $\beta$-amyloid plaques attract astrocytes and microglia, which lead to the production of proinflammatory cytokines such as interleukin-1 $\beta$, tumor necrosis factor- $\alpha$, and interferon- $\gamma$ (17).

Another important component of Alzheimer's disease pathology is the neurofibrillary tangles, which are composed of hyperphosphorylated $\tau$ proteins (18). Under normal conditions, $\tau$ proteins are involved as the components of microtubules, which aid in the transportation of cell objects to the end of axons. Therefore, it is likely that neurofibrillary tangles interfere with axonal transport, which is necessary for proper neuronal function, such as the transport of synaptic vesicles with neurotransmitters (19).

\section{Melanopsin-expressing RGCs}

mRGCs were identified when it was observed that mice with extensive degeneration of rods and cones were able to conduct normal circadian rhythms. Both blind and control subjects were found to have a light-induced melatonin suppression response, which suggested the presence of a different light perception system $(12,13,20)$.

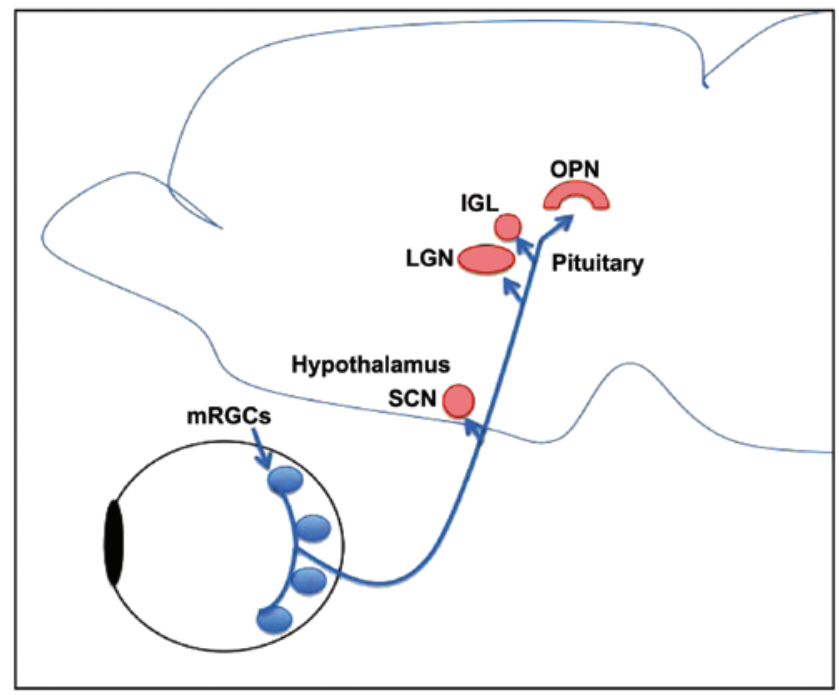

Figure 1. Diagrammatic representation of the pathway taken by melanopsin ganglion cells to the brain. The pathway takes the retinohypothalamic tract (blue pathway) to the suprachiasmatic nucleus, and then to the pituitary to regulate melatonin release. This circuit drives the circadian rhythm of the human body. SCN, suprachiasmatic nucleus; LGN, lateral geniculate nucleus; IGL, intergeniculate leaflet; mRGCs, melanopsin-expressing retinal ganglion cells; OPN, olivary pretectal nucleus.

The mRGCs, through the retinohypothalamic tract, regulate the non-vision forming functions of the eye such as circadian rhythms, pupil regulation, melatonin expression, cognition and sleep. mRGCs are intrinsically photosensitive due to the production of melanopsin even when there is no other retinal input. Melanopsin is a photopigment, which allows mRGCs to restore their chromophore, which is similar to the photoreceptors of plants. Thus, mRGCs absorb and process light as they are maximally sensitive to short-wavelength blue light. The mRGCs are also involved in developing the retina as these cells start functioning at day 0 post-birth. Besides sending messages to the suprachiasmatic nucleus, mRGCs also innervate to areas of the brain that are important to vision such as the locus coeruleus, lateral geniculate nucleus and superior colliculus $(12,21)$.

\section{The mRGCs in visual defects}

Blind subjects, in clinical trials, were found to have a preserved mRGCs-retinohypothalamic tract-suprachiasmatic nucleus circuit (22). As long as there is light perception present in blind subjects, this circuit is established. However, when the light perception is no longer present, the circuit disintegrates and the circadian rhythm is disturbed. The circadian rhythm is associated with the severity of the ocular disease, which affects the outer retina spare the mRGCs (23).

Certain disorders, including neurodegenerative optic neuropathies such as Leber hereditary optic neuropathy (LHON) and dominant optic atrophy (DOA), lead to the loss of RGCs. It was found that the subpopulations of mRGCs were resistant to neurodegeneration in these neuropathies while other RGCs were not resistant. Therefore, in patients with LHON and DOA, the circadian rhythm was preserved (24).

Glaucoma is another optic neuropathy that is characterized by loss of RGCs, leading to optic atrophy and blindness. The 
loss of mRGCs in animal models, due to glaucoma, leads to impairment of the circadian rhythms. In humans, glaucoma was found to correlate with the abnormal circadian rhythm of melatonin secretion and light-induced melatonin suppression in glaucoma patients. However, the pupillary light reflex is compromised in glaucoma patients. Therefore, the role of mRGCs in glaucoma has not been fully investigated and requires further clarification (25).

\section{Role of mRGCs in migraines}

Noseda et al (25) investigated the contribution of mRGCs to photophobia in migraines. Blind subjects with light perception maintain photophobia during migraines while blind subjects without light perception do not exhibit photophobia. The authors of that study found that mRGCs provided signals to the posterior thalamus, leading to mediation of photophobia in migraines (25). This is a major non-vision forming system of retina. The contribution of mRGCs to photophobia has also been documented in rodless and coneless mice (25).

\section{Role of mRGCs in aging}

Sleep is normally affected in the aging process due to abnormal homeostatic regulation. These changes are expressed as impaired non-rapid eye movement sleep by a decrease in slow wave activity, increased awakenings during the night and/or from circadian rhythm dysfunction. In addition, the elderly experience a decrease in the amplitude of circadian rhythms such as melatonin secretion, cortisol secretion and core body temperature. The phase advance of circadian rhythms, especially the sleep-wake cycle, and a decrease in circadian period was also observed in the elderly $(26,27)$.

There are various factors associated with the circadian dysfunction in the elderly. One of these factors includes neurodegenerative changes in the suprachiasmatic nucleus, such as a decrease in vasopressin and vasoactive intestinal peptide-expressing neurons. Another factor includes alterations in the pineal gland, which causes a decrease in the amplitude of the melatonin rhythm and expression of melatonin receptors. Another factor contributing to circadian dysfunction in the elderly is a decrease in light input to the suprachiasmatic nucleus due to a reduction in the capacity of the semicataractous lens to transmit short wavelength light (28). Other factors include degenerative changes in the retina and optic nerve with aging (29).

La Morgia et al (30) investigated the post-mortem retinal specimens in subjects that were in their mid-fifties and 1 subject that was $>80$ years. It was found that mRGCs, and regular RGCs, are lost with age. The mRGCs are distributed around the fovea and despite the loss in mRGCs, this distribution is maintained with age. Notably, while mRGCs are lost and impaired in the elderly, they remain robust and unaffected in optic neuropathies $(29,30)$.

\section{Role of mRGCs in Alzheimer's disease patients}

La Morgia et al (29) demonstrated a greater loss of mRGCs in Alzheimer's disease patients when compared to age-matched control patients. In addition, the remaining mRGCs that were still present in Alzheimer's disease patients presented morphological abnormalities, such as a reduction in dendritic diameter, which suggests functional impairment. Of note, Alzheimer's disease patients have a normal RGC count, but a significant decrease in $\mathrm{mRGC}$ count, which suggests that mRGCs are specifically modified in Alzheimer's disease. Furthermore, $\beta$-amyloid deposits were found post-mortem in Alzheimer's disease retinas, and they were found to specifically affect mRGCs (29).

Axonal loss and preferential loss of fibers in the superior quadrant has been documented in Alzheimer's disease patients. The superior quadrant contains the largest fibers of the optic nerve and postmortem studies of the Alzheimer's brain showed a loss of the larger axons with $\beta$-amyloid deposits. Axonal loss and pathology of the superior quadrant was similar to that of glaucoma and thus, Alzheimer's disease-induced optic neuropathy is similar to glaucoma (27).

\section{Changes in circadian rhythms in Alzheimer's disease patients}

Although there is a definite loss of mRCGs in Alzheimer's disease patients, the circadian rest-activity assessment is variable among the patients. This is most likely due to the pathology of Alzheimer's disease, which causes variable severity of the circadian rhythms in the patients. Although there were no significant differences between the circadian parameters of Alzheimer's disease and control patients, there was one subgroup of patients that had significantly different values (27). In addition, Alzheimer's disease patients were less active during the day and more active at night, suggesting a disturbance in circadian rhythms. The lack of difference in circadian values between Alzheimer's patients and control subjects may be due to the inclusion of mild-moderate Alzheimer's cases (5).

Other studies that have investigated at circadian patterns in Alzheimer's disease patients have found a correlation between severity of dementia and severity of circadian dysfunction. This change in results may be due to the inclusion of only moderate and severe cases. Across the board, Alzheimer's disease patients demonstrate decreased sleep efficiency and slow wave sleep amount, even in the mild cases (31).

In a follow-up study of Alzheimer's patients, Hatfield et al (5) showed that the circadian pattern, unlike cognition, did not deteriorate over time. This investigation demonstrated that cognitive and circadian decrease most likely do not progress at the same rate in individuals with Alzheimer's disease. However, the two were found to be correlated with each other and therefore, significant circadian dysfunction at the early stage of dementia can be used as a predictive factor of poor outcome in Alzheimer's disease patients.

When the Alzheimer's disease and age-matched control retinas examined at postmortem, the studies demonstrated that, not only was there total RGC loss, which correlated with aging, but there was also mRGC loss, which was specific to patients with Alzheimer's disease. In fact, younger Alzheimer's disease patients that had normal RGC count also demonstrated a significant loss in mRGCs. Therefore, in Alzheimer's disease, the mRGC subpopulation is sensitive to Alzheimer's disease neuropathological mechanisms (30).

A recent study (30) demonstrated that hyperphosphorylated $\tau$ deposits were present in the retinas of Alzheimer's 
disease patients. The $\beta$-amyloid deposits were also found inside and around mRGCs, which shows that mRGCs are primary retinal cells that are affected in Alzheimer's disease. An explanation for the specific loss of mRGCs may be retrograde axonal degeneration, which directs the pathology of suprachiasmatic nucleus. Alzheimer's disease neuropathology leads to neuronal loss in the suprachiasmatic nucleus and in the hypothalamic ventrolateral preoptic nucleus, the latter of which is correlated with sleep fragmentation. The considerable loss of mRGCs and the abnormality of the remaining mRGCs, in combination with the hypothalamic pathology in Alzheimer's disease, may contribute to sleep abnormality and circadian rhythm dysfunction $(12,26)$.

\section{Conclusion}

Alzheimer's disease pathogenesis establishes itself in not just cerebral blood vessels as well as optic vessels. Recent studies $(12,13,27)$ linking Alzheimer's disease to a new class of cells known as mRCGs has led to a new pathology for the loss of the circadian rhythm in patients with Alzheimer's disease. The hallmarks of Alzheimer's disease, which are $\beta$-amyloid deposits and neurofibrillary tangles, are present in the retinas of those with the disease. In addition, circadian dysfunction at early stage Alzheimer's disease was found to be a predictive factor for prognosis in Alzheimer's disease. More studies are required to determine the mechanism behind the loss of the circadian rhythm in Alzheimer's disease.

\section{References}

1. Arriagada PV, Growdon JH, Hedley-Whyte ET, Hyman BT: Neurofibrillary tangles but not senile plaques parallel duration and severity of Alzheimer's disease. Neurology 42: 631, 1992.

2. Bateman RJ, Xiong C, Benzinger TL, Fagan AM, Goate A, Fox NC, Marcus DS, Cairns NJ, Xie X, Blazey TM, et al; Dominantly Inherited Alzheimer Network: Clinical and biomarker changes in dominantly inherited Alzheimer's disease. N Engl J Med 367: 795-804, 2012.

3. Weiner MW, Veitch DP, Aisen PS, et al; Alzheimer's Disease Neuroimaging Initiative: The Alzheimer's Disease Neuroimaging Initiative: A review of papers published since its inception. Alzheimers Dement 8 (Suppl 1): S1-S68, 2012.

4. MishimaK,Okawa M,Hozumi S and Hishikawa Y: Supplementary administration of artificial bright light and melatonin as potent treatment for disorganized circadian rest-activity and dysfunctional autonomic and neuroendocrine systems in institutionalized demented elderly persons. Chronobiol Int 17: 419-432, 2000.

5. Hatfield CF, Herbert J, van Someren EJW, Hodges JR and Hastings MH: Disrupted daily activity/rest cycles in relation to daily cortisol rhythms of home-dwelling patients with early Alzheimer's dementia. Brain 127: 1061-1074, 2004.

6. Tranah GJ, Blackwell T, Stone KL, et al; SOF Research Group: Circadian activity rhythms and risk of incident dementia and mild cognitive impairment in older women. Ann Neurol 70: 722-732, 2011.

7. Sherman SM, Mumford JA and Schnyer DM: Hippocampal activity mediates the relationship between circadian activity rhythms and memory in older adults. Neuropsychologia 75 : 617-625, 2015.

8. Yoneda S, Hara H, Hirata A, Fukushima M, Inomata $\mathrm{Y}$ and Tanihara H: Vitreous fluid levels of $\beta$-amyloid (1-42) and tau in patients with retinal diseases. Jap J Ophthalmol: 106-108, 2005.

9. Ning A, Cui J, To E, Ashe KH and Matsubara J: Amyloid- $\beta$ deposits lead to retinal degeneration in a mouse model of Alzheimer disease. Invest Ophthalmol Vis Sci 49: 5136-5143, 2008.

10. Cotman SL, Halfter W and Cole GJ: Agrin binds to $\beta$-amyloid (A $\beta)$, accelerates $A \beta$ fibril formation, and is localized to $A \beta$ deposits in Alzheimer's disease brain. Mol Cell Neurosci 15: 183-198, 2000.
11. Koronyo-Hamaoui M, Koronyo Y, Ljubimov AV, Miller CA, Ko MK, Black KL, Schwartz M and Farkas DL: Identification of amyloid plaques in retinas from Alzheimer's patients and noninvasive in vivo optical imaging of retinal plaques in a mouse model. Neuroimage 54 (Suppl 1): S204-S217, 2011.

12. LeGates TA, Altimus CM, Yang S, Kirkwood A, Weber ET and Hattar S: Melanopsin-expressing retinal ganglion cells mediate light modulation of cognitive functions and mood related behaviors. Invest Ophthalmol Vis Sci 52: 3465, 2011.

13. Sosa-Ortiz AL, Acosta-Castillo I and Prince MJ: Epidemiology of dementias and Alzheimer's disease. Arch Med Res 43: 600-608, 2012

14. Lopez AD, Mathers CD, Ezzati M, Jamison DT and Murray CJ: Global and regional burden of disease and risk factors, 2001: systematic analysis of population health data. Lancet 367 : $1747-1757,2006$

15. Karran E, Mercken M and De Strooper B: The amyloid cascade hypothesis for Alzheimer's disease: An appraisal for the development of therapeutics. Nat Rev Drug Discov 10: 698-712, 2011.

16. De Strooper B, Vassar R and Golde T: The secretases:enzymes with therapeutic potential in Alzheimer disease. Nat Rev Neurol 6: 99-107, 2010.

17. de Calignon A, Polydoro M, Suárez-Calvet M, William C, Adamowicz DH, Kopeikina KJ, Pitstick R, Sahara N, Ashe KH, Carlson GA, et al: Propagation of tau pathology in a model of early Alzheimer's disease. Neuron 73: 685-697, 2012.

18. Bancher C, Brunner C, Lassmann H, Budka H, Jellinger K, Wiche G, Seitelberger F, Grundke-Iqbal I, Iqbal K and Wisniewski HM: Accumulation of abnormally phosphorylated $\tau$ precedes the formation of neurofibrillary tangles in Alzheimer's disease. Brain Res 477: 90-99, 1989.

19. LeGates TA, Altimus CM, Wang H, Lee HK, Yang S, Zhao H, Kirkwood A, Weber ET and Hattar S: Aberrant light directly impairs mood and learning through melanopsin-expressing neurons. Nature 491: 594-598, 2012.

20. Cui Q, Ren C, Sollars PJ, Pickard GE and So KF: The injury resistant ability of melanopsin-expressing intrinsically photosensitive retinal ganglion cells. Neuroscience 284: 845-853, 2015.

21. González Fleitas MF, Bordone M, Rosenstein RE and Dorfman D: Effect of retinal ischemia on the non-image forming visual system. Chronobiol Int 32: 152-163, 2015.

22. Czeisler CA, Shanahan TL, Klerman EB, Martens H, Brotman DJ, Emens JS, Klein T and Rizzo JF: Suppression of melatonin secretion in some blind patients by exposure to bright light. N Engl J Med 332: 6-11, 1995.

23. Moura AL, Nagy BV, La Morgia C, Barboni P, Oliveira AG, Salomão SR, Berezovsky A, de Moraes-Filho MN, Chicani CF, Belfort R Jr, et al: The pupil light reflex in Leber's hereditary optic neuropathy: Evidence for preservation of melanopsin-expressing retinal ganglion cells. Invest Ophthalmol Vis Sci 54: 4471-4477, 2013.

24. de Zavalía N, Plano SA, Fernandez DC, Lanzani MF, Salido E, Belforte N, Sarmiento MI, Golombek DA and Rosenstein RE: Effect of experimental glaucoma on the non-image forming visual system. J Neurochem 117: 904-914, 2011.

25. Noseda R, Kainz V, Jakubowski M, Gooley JJ, Saper CB, Digre K and Burstein R: A neural mechanism for exacerbation of headache by light. Nat Neurosci 13: 239-245, 2010.

26. Ross-Cisneros F, La Morgia C, Pan B, Hannibal J, Carelli V and Sadun A: A Histopathologic and morphometric analysis of degenerating melanopsin retinal ganglion cells in Alzheimer's disease. Invest Ophthalmol Vis Sci 54: 299, 2013.

27. van Someren EJ, Hagebeuk EE, Lijzenga C, Scheltens P, de Rooij SE, Jonker C, Pot AM, Mirmiran M and Swaab DF: Circadian rest-activity rhythm disturbances in Alzheimer's disease. Biol Psychiatry 40: 259-270, 1996.

28. Hofman MA and Swaab DF: Living by the clock: the circadian pacemaker in older people. Ageing Res Rev 5: 33-51, 2006.

29. La Morgia C, Gallassi R, Sambati L, Provini F, Ross-Cisneros FN, Pan B, Barboni P, et al: Melanopsin retinal ganglion cells and circadian dysfunction in Alzheimer's disease. Acta Ophthalmologica 91: 0, 2013.

30. La Morgia C, Ross-Cisneros FN, Koronyo Y, Hannibal J, Gallassi R, Cantalupo G, Sambati L, Pan BX, Tozer KR, Barboni P, et al: Melanopsin retinal ganglion cell loss in Alzheimer's disease. Ann Neurol: Oct 27, 2015 (Epub ahead of print).

31. Dai J, Swaab DF, Van der Vliet J and Buijs RM: Postmortem tracing reveals the organization of hypothalamic projections of the suprachiasmatic nucleus in the human brain. J Comp Neurol 400: 87-102, 1998. 